\title{
Métodos de mensuração do letramento em saúde bucal no Brasil: uma revisão integrativa
}

\author{
Measurement methods of oral health literacy in Brazil: an integrative review
}
Métodos de medición de la alfabetización en salud bucal en Brasil: una revisión integradora

Renata Matos Lamenha Lins ${ }^{1 *}$, Maria Clara Cavalcanti Campêlo ${ }^{1}$, Leopoldo Cosme Silva ${ }^{1}$, João Victor Farias da Silva ${ }^{2}$, Cristine D'Almeida Borges ${ }^{1}$, Ana Regina Oliveira Moreira ${ }^{1}$, Pamella Recco Alvares $^{3}$, Priscylla Gonçalves Correia Leite de Marcelos ${ }^{1}$, Daniela Maria Carvalho Pugliesi ${ }^{1}$, Valdeci Elias dos Santos Júnior ${ }^{1}$.

\begin{abstract}
RESUMO
Objetivo: Identificar e avaliar criticamente os atuais métodos validados para a mensuração do Letramento em Saúde Bucal (LSB) no Brasil, bem como seus achados epidemiológicos. Métodos: A busca desta revisão integrativa da literatura foi conduzida em sete bases de dados. Os critérios de elegibilidade abrangeram estudos epidemiológicos conduzidos no Brasil, de validação de instrumentos para o português brasileiro e avaliação das propriedades psicométricas das ferramentas. Todo o processo de exclusão e inclusão seguiu as recomendações do Preferred Reporting Items for Systematic Reviews and Meta-Analyses (PRISMA). Resultados: Dez artigos foram incluídos neste estudo. Foi verificado que apenas quatro instrumentos de mensuração do LSB possuem validação para uso no Brasil, delineados com diferentes propósitos e propriedades psicométricas. A maioria dos estudos epidemiológicos foi conduzida na região Sul do país (60\%) e não foram encontradas pesquisas nas regiões Norte e Centro-Oeste. Considerações Finais: Futuros estudos epidemiológicos que utilizem os instrumentos recentemente validados para a mensuração do grau de LSB, em uma disposição demográfica mais proporcional, são fundamentais para que possam ser identificadas falhas nos métodos de educação em saúde atualmente empregados, aprimorando as políticas de saúde bucal e permitindo ao clínico/pesquisador uma intervenção mais próxima da realidade da população na qual atua.
\end{abstract}

Palavras-chave: Educação em saúde bucal, Saúde bucal, Saúde da população, Política de saúde.

\begin{abstract}
Objective: Identify and critically analyze current validated methods for Oral Health Literacy (OHL) measurement in Brazil, as well as its related epidemiological findings. Methods: The literature research of this integrative review was conducted in seven databases. Eligibility criteria included epidemiological studies conducted in Brazil, articles of instruments' validation to Brazilian Portuguese and assessment of its psychometric properties. The inclusion and exclusion process followed the recommendations of the Preferred Reporting Items for Systematic Reviews and Meta-Analyses (PRISMA). Pertinent information was condensed, and a critical analysis of the tools and their epidemiological researches was performed. Results: Ten articles were included in this study. It was verified that only four instruments of $\mathrm{OHL}$ measurement present validation for use in Brazil, designed with different purposes and psychometric properties. Most of the epidemiological studies were conducted in the South region of the country $(60 \%)$ and researches in Northern and Midwest regions were not found. Final Considerations: Future epidemiological studies utilizing the recently validated instruments for the measurement of $\mathrm{OHL}$, according to a more proportional demographic arrangement, are fundamental to identify failures in the health education methods currently applied, improving oral health policies and providing a closer intervention of the clinician/researcher to the population surveyed.
\end{abstract}

Keywords: Dental health education, Oral health, Population health, Health policy.

\footnotetext{
${ }^{1}$ Universidade Federal de Alagoas (UFAL), Maceió - AL. *E-mail: renatamlamenha@gmail.com

2 Universidade Federal de Sergipe (UFS), Aracaju - SE.

${ }^{3}$ Universidade de Pernambuco (UPE), Recife - PE.
} 


\section{RESUMEN}

Objetivo: Identificar y evaluar críticamente los métodos validados actuales para medir la Alfabetización en Salud Bucal (LSB) en Brasil, así como sus hallazgos epidemiológicos. Métodos: La búsqueda de esta revisión integradora de la literatura se realizó en siete bases de datos. Los criterios de elegibilidad incluyeron estudios epidemiológicos realizados en Brasil, validación de instrumentos para portugués brasileño y evaluación de las propiedades psicométricas de las herramientas. Todo el proceso de exclusión e inclusión siguió la recomendación de los elementos de informe Preferidos para revisiones sistemáticas y metanálisis (PRISMA). Resultados: Se incluyeron diez artículos en este estudio. Se encontró que solo cuatro instrumentos de medición LSB tienen validación para su uso en Brasil, diseñados con diferentes propósitos y propiedades psicométricas. La mayoría de los estudios epidemiológicos se realizaron en la región sur del país $(60 \%)$ y no se encontraron en las regiones Norte y Centro Oeste. Consideraciones Finales: Los estudios epidemiológicos futuros que utilicen los instrumentos recientemente validados para medir el grado de LSB, en una disposición demográfica más proporcional, son fundamentales para que puedan ser identificados en los métodos de educación para la salud actualmente empleados, mejorando las políticas de salud bucodental y permitiendo al clínico / investigador una intervención más cercana a la realidad de la población en la que trabaja.

Palabras clave: Educación en salud dental, Salud bucal, Salud poblacional, Política de salud.

\section{INTRODUÇÃO}

No Brasil, segundo o Indicador de Analfabetismo Funcional, 29\% dos jovens e adultos entre 15 e 64 anos apresentam dificuldade ou são incapazes de aplicar a leitura, escrita e numerácia em situações do cotidiano como, por exemplo, reconhecer informações em panfletos (IPM, 2018).

De acordo com a Organização Mundial de Saúde, inevitavelmente, o nível de alfabetização do indivíduo influencia na sua capacidade de letramento em saúde e atua como um forte preditor da renda, situação de emprego e nível de educação (WHO, 2013). Nessa perspectiva, os aspectos de origem social, económica, cultural, ambiental e biológica/genética atuam como determinantes da saúde do indivíduo (CARRAPATO P, et al., 2017).

O letramento em saúde refere-se à representação das competências cognitivas e sociais as quais determinam a motivação e habilidade dos indivíduos em adquirir acesso, compreensão e utilização de informações de maneira a promover e manter sua boa saúde geral, bem como empoderamento a nível pessoal e comunitário (WHO, 2013).

Definido desta maneira, é evidente a sua atuação como possível limitador do acesso aos serviços de saúde, a ser superado pelo indivíduo, profissionais da área e políticas de saúde pública. Baixos indicadores de letramento em saúde têm demonstrado resultar em comportamentos individuais e coletivos de risco, piores condições de saúde, menor poder de autogerenciamento e maiores índices de hospitalização, aumentando substancialmente os custos dos serviços públicos (WHO, 2010).

A prevalência da cárie dentária no Brasil permanece elevada e enfaticamente polarizada e, portanto, representa um desafiante problema de saúde pública (BRASIL, 2012). Tal fato, associado à inter-relação entre os menores índices de letramento em saúde e desfechos clínicos prejudiciais na área médica, incentivou a recente investigação do nível de letramento no campo da saúde bucal e craniofacial no país (NIDCR, et al., 2005).

A mensuração do nível de letramento em saúde bucal (LSB) do indivíduo, por intermédio de instrumentos validados específicos para os cuidados odontológicos, possibilita a identificação de lacunas relacionadas à promoção de saúde bucal e beneficia a comunicação profissional-paciente (NIDCR, et al., 2005; SORENSEN K, et al., 2005; DICKSON-SWIFT V, et al., 2014; PARTHASARATHY DS, et al., 2014). Para tal, a análise das propriedades psicométricas de cada ferramenta disponível se faz necessária, de maneira que possam ser apropriadamente selecionadas de acordo com o objetivo da investigação epidemiológica (PARTHASARATHY DS, et al., 2014). 
Embora o LSB represente uma área atualmente emergente na comunidade científica e possua destacável relevância para estudos epidemiológicos e políticas de saúde pública, a sua recente inserção nos campos de pesquisa por meio da validação de instrumentos para o português brasileiro dificulta a compreensão e ampliação do seu uso pelo clínico ou pesquisador (SIMONDS SK, 1974; NIDCR, et al., 2005).

Dessa forma, esta revisão integrativa da literatura teve como objetivo identificar e avaliar criticamente os atuais métodos validados para a mensuração do Letramento em Saúde Bucal no Brasil, bem como seus achados epidemiológicos relacionados.

\section{MÉTODOS}

A fim de possibilitar a síntese do conhecimento a respeito dos atuais métodos para a mensuração do letramento em saúde bucal (LSB) no Brasil e apontar possíveis lacunas científicas a serem abordadas em futuros estudos, uma revisão integrativa da literatura foi realizada, utilizando-se a metodologia atualizada de Whittemore R e Knafl K (2005). Assim, uma busca compreensiva da literatura foi conduzida e, então, informações pertinentes dos estudos foram revisadas e condensadas.

\section{Critérios de elegibilidade}

Uma vez que havia a necessidade de uma maior abrangência de cobertura para a identificação dos dados, uma pergunta ampla contendo os termos centrais da busca foi elaborada, gerando a questão norteadora: "Quais instrumentos de letramento em saúde bucal validados são atualmente utilizados em estudos epidemiológicos no Brasil?".

Nesse contexto, corresponderam aos critérios de inclusão desta revisão: artigos publicados em periódicos que passaram por critério de revisão por pares e pesquisas epidemiológicas (estudos transversais, controle de caso, coorte e ensaios clínicos randomizados) as quais utilizaram instrumentos de avaliação do letramento em saúde bucal previamente validados para o português do Brasil. Ainda, foram incluídos artigos de validação de métodos de mensuração do LSB para aplicação em território nacional e estudos de análise das propriedades psicométricas de tais ferramentas.

Foram excluídos desta revisão resultados duplicados em diferentes plataformas de busca, relatos de caso, série de casos, cartas ao editor, resumos publicados em anais de congressos, dissertações, teses, revisões de literatura, estudos envolvendo letramento em saúde não específico para a Odontologia ou que utilizaram instrumentos não validados para uso no Brasil, bem como que não foram conduzidos em indivíduos que possuíam como idioma nativo o português brasileiro.

\section{Estratégia de busca}

A busca foi conduzida de Janeiro a Março de 2020 em ambas as línguas Português e Inglês, a fim de alcançar a intensa demanda de publicações internacionais dos trabalhos realizados no Brasil. Foram utilizadas as bases de dados Cochrane Library, US National Library of Medicine (PubMed), Web of Science, Scientific Electronic Library Online (SciELO), Literatura Latino-Americana e do Caribe em Ciências da Saúde (Lilacs), Medical Literature Analysis and Retrieval System Online (MEDLINE) e Bibliografia Brasileira de Odontologia (BBO), por intermédio da Biblioteca Virtual em Saúde (Bireme, América Latina).

A busca eletrônica combinou descritores MeSH (Medical Subject Headings) e DeCS (Descritores em Ciências da Saúde) com palavras-chave, intercalados entre si com a utilização de operadores booleanos. $O$ intervalo de publicação dos estudos não foi limitado, em virtude da incipiência de pesquisas sobre o tema.

Nas bases Cochrane Library e PubMed, foram utilizadas as estratégias de busca: ("Oral health literacy" OR "literacy" NEAR "dentistry" OR "literacy" NEAR "oral health" AND Brazil); (("health literacy" [MeSH] OR "health literacy" OR "oral health literacy" OR "literacy in dentistry") AND "Brazi*"). Nas plataformas Web of Science e SciELO, foram utilizadas as buscas avançadas ("oral health literacy" AND Brazil*); ("health literacy" $A N D$ "Brazi*”); (("alfabetização em saúde" OR "letramento em saúde" OR "alfabetismo em saúde") AND $\left(\right.$ Brasil $\left.^{*}\right)$. 
Utilizando-se a Biblioteca Virtual em Saúde, a estratégia de busca correspondeu à combinação do DeCS "alfabetização em saúde" com o termo livre "Brasil", relacionados entre si com o operador booleano AND, para as plataformas Lilacs, MEDLINE e BBO.

Apesar do descritor empregado na busca não ser referido estritamente ao letramento em saúde relacionado à Odontologia, seu uso justifica-se em consequência da necessidade de um maior alcance de dados, bem como devido às amplas variações existentes na língua portuguesa associadas ao letramento em saúde. Comumente, a literatura científica brasileira aborda a tradução do termo "health literacy" como "letramento em saúde", "alfabetização em saúde", "letramento funcional em saúde" e "literácia em saúde".

O software Reference Manager® (Reference Manager, Thomson Reuteurs, versão 12.0.3, Nova York, NY, EUA) foi empregado para identificar os estudos duplicados, assim como ordenar a lista de resumos dos artigos rastreados, para possibilitar uma avaliação mais criteriosa.

Todo o processo de exclusão e inclusão desta revisão seguiu as recomendações do Preferred Reporting Items for Systematic Reviews and Meta-Analyses - PRISMA, como também a transparência no relato dos resultados, utilizando-se um fluxograma (HUTTON B, et al., 2015).

\section{Análise dos dados}

Uma vez que métodos específicos para a avaliação comparativa e qualitativa dos instrumentos de letramento em saúde não existem, esta revisão não fez uso de ferramentas para tal análise (STONBRAKER S, et al., 2015).

A ampla gama de propriedades psicométricas a serem exploradas para envolver o constructo em questão faz com que cada ferramenta possua um diferente direcionamento para a avaliação da literácia, podendo, assim, ser abordados seus aspectos básico/funcional, comunicativo/interativo ou crítico/ativo (NUTBEAM D, 2008). Tais características tornam seu processo de qualificação por meio de escalas complexo e, portanto, inviável (STONBRAKER S, et al., 2015).

Dessa forma, este estudo estabeleceu uma comparação crítica das propriedades psicométricas dos instrumentos atualmente validados no Brasil, sendo ordenado: o nome da ferramenta, a habilidade de letramento analisada para a mensuração do LSB, número de itens presentes, margem de pontuação, validade e confiabilidade.

Além disso, foi esquematizada a visão cronológica geral dos artigos epidemiológicos incluídos nesta revisão, relacionando-se: a referência e delineamento do estudo, seu objetivo, tamanho e tipo da amostra avaliada, instrumento de LSB utilizado, principais resultados relacionados a determinantes socioeconômicos e/ou desfechos clínicos e as limitações da pesquisa. Os achados epidemiológicos foram analisados quanto ao instrumento empregado para a mensuração do LSB, distribuição em território nacional e população amostral estudada.

\section{RESULTADOS}

O total de artigos encontrados, somando-se todas as plataformas de busca, foi 377 . Inicialmente, os artigos duplicados foram excluídos $(n=98)$ e os estudos restantes $(n=279)$ foram analisados de acordo com o título, resumo e palavras-chave.

Artigos que não se enquadravam no escopo deste estudo foram excluídos $(n=251)$ e os demais foram lidos integralmente para que a elegibilidade fosse certificada, permanecendo, então, 10 artigos selecionados por intermédio das estratégias de busca. A Figura 1 esquematiza, em um fluxograma, o processo de exclusão e inclusão desta revisão integrativa, de acordo com as diretrizes do PRISMA (HUTTON B, et al., 2015). 
Figura 1 - Fluxograma PRISMA do processo de exclusão e inclusão da revisão integrativa.
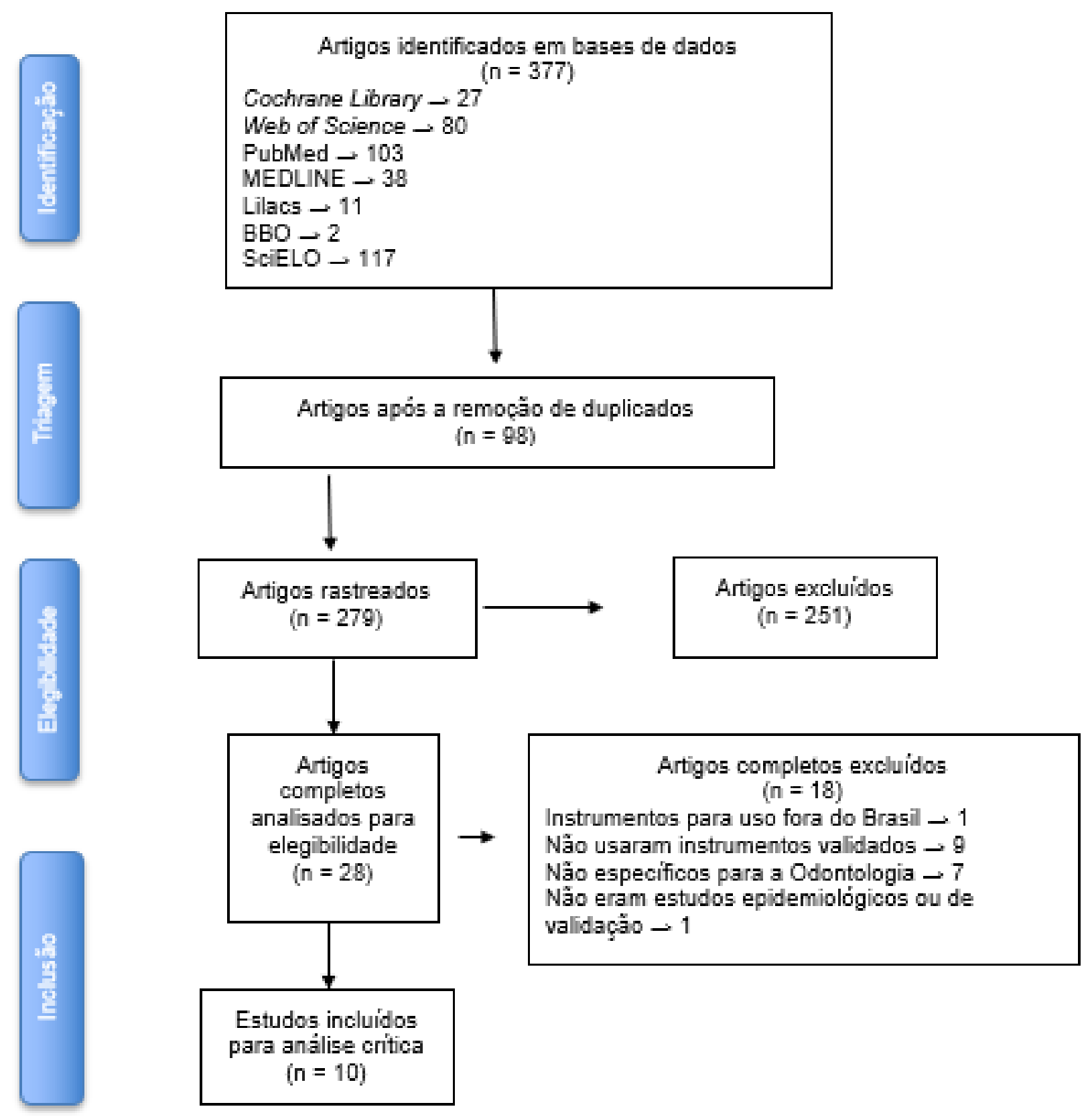

Legenda: PRISMA: Preferred Reporting Items for Systematic Reviews and Meta-Analyses. PubMed: US National Library of Medicine; MEDLINE: Medical Literature Analysis and Retrieval System Online; Lilacs: Literatura Latino-Americana e do Caribe em Ciências da Saúde; BBO: Bibliografia Brasileira de Odontologia; SciELO: Scientific Electronic Library Online. Fonte: Lins RML, et al., 2020.

Foi verificado que apenas quatro instrumentos para a mensuração do letramento em saúde bucal estão disponíveis validados para uso no Brasil, sendo estes: Brazilian Rapid Estimate of Adult Literacy in Dentistry (BREALD-30), Rapid Estimate Adult Literacy in Medicine and Dentistry (REALMD-20), Oral Health Literacy Assessment-Brazilian (OHLA-B) e Brazilian Hong Kong Oral Health Literacy Assessment Task for Pediatric Dentistry (BHKOHLAT-P) (JUNKES MC, et al., 2015; CRUVINEL AFP, et al., 2017; BADO FMR, et al., 2018; FIRMINO RT, et al., 2020). Foram dispostas as propriedades psicométricas de cada instrumento disponível, sendo analisados: o tipo de habilidade em letramento avaliada, número de itens presentes, margem de pontuação atribuída, validade e confiabilidade (Tabela 1). 
Tabela 1 - Propriedades psicométricas dos instrumentos validados para o português brasileiro.

\begin{tabular}{|c|c|c|c|c|c|}
\hline Instrumento & $\begin{array}{l}\text { Habilidade } \\
\text { avaliada }\end{array}$ & Itens & Pontuação & Validade & Confiabilidade \\
\hline BREALD-30 & $\begin{array}{l}\text { Reconhecimento } \\
\text { de palavras }\end{array}$ & 30 & $0-30$ & $\begin{array}{c}\text { CV: INAF }(r=0.593 ; \\
\left.p<0.001^{*}\right), \text { escolaridade } \\
\left(r=0.541 ; p<0.001^{*}\right) ; \\
\text { DV: ocupação }\left(p=0.004^{* *}\right) \\
\text { visita odontológica } \\
\left(p=0.017^{* *}\right) \text { e renda familiar } \\
\text { mensal }\left(p<0.001^{* *}\right)\end{array}$ & $\begin{array}{c}\alpha=0.88 \\
\mathrm{CCl}=0.98\end{array}$ \\
\hline REALMD-20 & $\begin{array}{l}\text { Reconhecimento } \\
\text { de palavras }\end{array}$ & 20 & $0-20$ & $\begin{array}{c}\text { CV: BREALD-30 }(r=0.73 ; \\
\text { p<0.001*), INAF }(r=0.60 ; \\
\left.p<0.001^{*}\right) ; \\
\text { DV: educação }\left(p<0.001^{* *}\right), \\
\text { ocupação }\left(p<0.001^{* *}\right), \text { auto } \\
\text { relato de saúde bucal } \\
\left(p<0.001^{* *}\right) \text { e razão de } \\
\text { consulta odontológica } \\
\left(p=0.01^{* *}\right)\end{array}$ & $\begin{array}{l}\alpha=0.789 \\
\mathrm{CCl}=0.73\end{array}$ \\
\hline OHLA-B & $\begin{array}{l}\text { Reconhecimento e } \\
\text { compreensão de } \\
\text { palavras }\end{array}$ & 15 & $0-15$ & $\begin{array}{c}\text { CT: Análise fatorial } \\
\text { exploratória (Kayser-Meyer- } \\
\text { Olkin = 0.78; Teste de } \\
\text { esfericidade de Bartlett }= \\
563.9 \text { ); } \\
\text { Análise fatorial confirmatória } \\
\text { (Índice de ajuste } \\
\text { comparativo }=0.97 ; \text { Erro } \\
\text { quadrático médio de } \\
\text { aproximação }=0.06 \text { ) }\end{array}$ & $\begin{array}{l}\alpha=0.83 \\
\omega t=0.80\end{array}$ \\
\hline BHKOHLAT-P & $\begin{array}{l}\text { Conhecimento em } \\
\text { saúde bucal, } \\
\text { compreensão de } \\
\text { leitura e numerácia }\end{array}$ & 49 & $0-49$ & $\begin{array}{l}\text { CV: BREALD-30 }(r=0.704 ; \\
\left.p<0.001^{*}\right), \text { escolaridade } \\
\left(r=0.602 ; p<0.001^{*}\right), \text { horas } \\
\text { de leitura }(r=0.342 ; \\
\left.p<0.001^{\star}\right) ; \\
\text { DV: cárie dentária do filho } \\
\left(p<0.005^{\star *}\right) . \text { Em modelo } \\
\text { multivariado, a associação } \\
\text { perdeu a significância } \\
\text { estatística. }\end{array}$ & $\begin{array}{c}\alpha=0.92 \\
\mathrm{CCl}=0.95\end{array}$ \\
\hline
\end{tabular}

Legenda: *Coeficiente de correlação de Spearman; ${ }^{*}$ Teste de Mann-Whitney. a: Coeficiente Alfa de Cronbach; CCI: Coeficiente de correlação intra-classe; wt: Coeficiente ômega de McDonald. CV: Validade convergente; DV: Validade divergente; CT: Validade de constructo. INAF: Indicador Nacional de Analfabetismo Funcional. BREALD-30: Brazilian Rapid Estimate of Adult Literacy in Dentistry; REALMD-20: Rapid Estimate Adult Literacy in Medicine and Dentistry; OHLA-B: Oral Health Literacy Assessment-Brazilian; BHKOHLAT-P: Brazilian Hong Kong Oral Health Literacy Assessment Task for Pediatric Dentistry.

Fonte: Lins RML, et al., 2020.

O BREALD-30 demonstrou ser a ferramenta mais utilizada em território nacional. Foi relacionada a visão geral dos artigos epidemiológicos envolvendo o letramento em saúde bucal no território brasileiro, apresentando seu ano de publicação, objetivo, tamanho e tipo da amostra, instrumento utilizado para a mensuração, principais resultados atrelados a determinantes socioeconômicos e/ou desfechos clínicos e as limitações do estudo (JUNKES MC, et al., 2015; VILELLA KD, et al., 2016; VILELLA KD, et al., 2017; CRUVINEL AFP, et al., 2017; BARASUOL JC, et al., 2017; FIRMINO RT, et al., 2018; MONTES GR, et al., 2019; LIMA LCM, et al., 2019; FIRMINO RT, et al., 2020) (Quadro 1).

A maioria dos estudos selecionados $(60 \%)$ foi conduzida na região Sul do país, enquanto $20 \%$ se desenvolveram na região Sudeste e $20 \%$ na região Nordeste. Não foram identificados quaisquer estudos envolvendo a mensuração do LSB nas regiões Norte e Centro-Oeste. 


\section{Revista Eletrônica Acervo Saúde / Electronic Journal Collection Health | ISSN 2178-2091}

Quadro 1 - Visão cronológica geral dos estudos epidemiológicos utilizando instrumentos de mensuração do letramento em saúde bucal (LSB) no Brasil.

\begin{tabular}{|c|c|c|c|c|c|}
\hline $\begin{array}{l}\text { Referência e } \\
\text { delineamento }\end{array}$ & Objetivo & Amostra & $\begin{array}{l}\text { Ferramenta } \\
\text { utilizada }\end{array}$ & $\begin{array}{c}\text { Principais resultados atrelados a } \\
\text { determinantes socioeconômicos e/ou } \\
\text { desfechos clínicos }\end{array}$ & Limitações \\
\hline $\begin{array}{l}\text { JUNKES MC, et al., } \\
\quad 2015 ; \\
\text { Estudo transversal }\end{array}$ & $\begin{array}{c}\text { Traduzir, realizar a } \\
\text { adaptação transcultural do } \\
\text { REALD e verificar a } \\
\text { confiabilidade e validade do } \\
\text { instrumento }\end{array}$ & $\begin{array}{l}258 \text { adultos } \\
\text { cuidadores de } \\
\text { crianças, em clínica } \\
\text { odontológica }\end{array}$ & BREALD-30 & $\begin{array}{c}\text { A pontuação do BREALD-30 foi } \\
\text { estatisticamente correlacionada com o nível } \\
\text { de literácia geral e renda; e associada com a } \\
\text { ocupação, escolaridade, uso de serviços } \\
\text { odontológicos, auto-avaliação de saúde bucal } \\
\text { e a percepção do respondente quanto à } \\
\text { saúde bucal do seu filho }\end{array}$ & $\begin{array}{l}\text { Amostragem não-aleatória e } \\
\text { predominantemente do sexo } \\
\text { feminino; Análise de apenas } \\
\text { uma habilidade relativa à } \\
\text { literácia }\end{array}$ \\
\hline $\begin{array}{l}\text { VILELLA KD, et al., 2016; } \\
\text { Estudo transversal }\end{array}$ & $\begin{array}{l}\text { Avaliar o LSB de mulheres } \\
\text { grávidas e a sua associação } \\
\text { com determinantes sociais e } \\
\text { conhecimento de hábitos } \\
\text { alimentares e higiene bucal } \\
\text { em crianças }\end{array}$ & $\begin{array}{l}175 \text { mulheres } \\
\text { grávidas, na } \\
\text { maternidade de um } \\
\text { hospital universitário }\end{array}$ & BREALD-30 & $\begin{array}{c}\text { O grau de LSB foi positivamente } \\
\text { correlacionado com o conhecimento em } \\
\text { saúde bucal e a idade na qual a mãe } \\
\text { pretendia introduzir açúcar na dieta do filho; e } \\
\text { uma correlação negativa foi identificada com a } \\
\text { aglomeração domiciliar. O alto grau de LSB } \\
\text { foi associado com anos de escolaridade, } \\
\text { maior classe socioeconômica e possuir } \\
\text { emprego }\end{array}$ & $\begin{array}{l}\text { Amostragem não-aleatória; } \\
\text { Análise de apenas uma } \\
\text { habilidade relativa à literácia }\end{array}$ \\
\hline $\begin{array}{l}\text { VILELLA KD, et al., 2017; } \\
\text { Ensaio clínico } \\
\text { randomizado }\end{array}$ & $\begin{array}{l}\text { Avaliar o efeito do LSB na } \\
\text { retenção de informação em } \\
\text { saúde em mulheres grávidas }\end{array}$ & $\begin{array}{l}162 \text { mulheres } \\
\text { grávidas registradas } \\
\text { em programa pré- } \\
\text { natal }\end{array}$ & BREALD-30 & $\begin{array}{l}\text { Mulheres grávidas com baixo grau de LSB } \\
\text { demonstraram menor conhecimento } \\
\text { relacionado aos hábitos alimentares e higiene } \\
\text { bucal das crianças em todos os tipos de } \\
\text { intervenção para educação em saúde: escrita, } \\
\text { falada e controle }\end{array}$ & $\begin{array}{l}\text { Amostragem não-aleatória e } \\
\text { proveniente do mesmo } \\
\text { centro; Análise de apenas } \\
\text { uma habilidade relativa à } \\
\text { literácia }\end{array}$ \\
\hline $\begin{array}{l}\text { CRUVINEL AFP, et al., } \\
2017 ; \\
\text { Estudo transversal }\end{array}$ & $\begin{array}{l}\text { Performar a adaptação } \\
\text { transcultural e validação do } \\
\text { REALMD-20 para pacientes } \\
\text { odontológicos brasileiros }\end{array}$ & $\begin{array}{l}200 \text { adultos, em } \\
\text { clínica odontológica }\end{array}$ & $\begin{array}{l}\text { REALMD-20 } \\
\text { BREALD-30 }\end{array}$ & $\begin{array}{c}\text { A pontuação do REALMD-20 foi } \\
\text { significativamente maior entre profissionais da } \\
\text { saúde, pessoas com maiores níveis de } \\
\text { educação, que relataram boa/excelente saúde } \\
\text { bucal e buscavam por atendimento } \\
\text { odontológico preventivo. O LSB foi preditor } \\
\text { significativo da condição auto-declarada de } \\
\text { saúde bucal }\end{array}$ & $\begin{array}{l}\text { Amostragem não reflete a } \\
\text { estrutura demográfica } \\
\text { genuína brasileira; Método } \\
\text { discutível para estudos } \\
\text { longitudinais; Validação } \\
\text { preditiva apenas com } \\
\text { desfechos dentários }\end{array}$ \\
\hline $\begin{array}{l}\text { BARASUOL JC, et al., } \\
2017 ; \\
\text { Estudo transversal }\end{array}$ & $\begin{array}{l}\text { Avaliar a associação entre a } \\
\text { ansiedade relacionada ao } \\
\text { tratamento dentário dos pais } \\
\text { e variáveis independentes }\end{array}$ & $\begin{array}{l}168 \text { adultos } \\
\text { cuidadores de } \\
\text { crianças, em clínica } \\
\text { odontológica }\end{array}$ & BREALD-30 & $\begin{array}{l}\text { Altos níveis de ansiedade relacionada ao } \\
\text { tratamento dentário dos pais foram } \\
\text { associados à renda familiar igual ou inferior a } \\
\text { um salário mínimo e ao menor nível de LSB }\end{array}$ & $\begin{array}{l}\text { Amostragem não-aleatória; } \\
\text { Possibilidade de viés de } \\
\text { interpretação dos } \\
\text { questionários aplicados }\end{array}$ \\
\hline
\end{tabular}

REAS / EJCH | Vol.12(12) | e4993 | DOI: https://doi.org/10.25248/reas.e4993.2020 Página 7 de 12 
Revista Eletrônica Acervo Saúde / Electronic Journal Collection Health | ISSN 2178-2091

\begin{tabular}{|c|c|c|c|c|c|}
\hline $\begin{array}{c}\text { Referência e } \\
\text { delineamento }\end{array}$ & Objetivo & Amostra & $\begin{array}{l}\text { Ferramenta } \\
\text { utilizada }\end{array}$ & $\begin{array}{c}\text { Principais resultados atrelados a } \\
\text { determinantes socioeconômicos e/ou } \\
\text { desfechos clínicos }\end{array}$ & Limitações \\
\hline $\begin{array}{l}\text { FIRMINO RT, et al., } \\
\quad 2018 \\
\text { Estudo transversal }\end{array}$ & $\begin{array}{l}\text { Investigar se o LSB afeta a } \\
\text { ausência de dados obtidos } \\
\text { por meio de auto-relato em } \\
\text { pesquisa epidemiológica em } \\
\text { saúde bucal }\end{array}$ & $\begin{array}{c}344 \text { adultos } \\
\text { cuidadores de } \\
\text { crianças pré- } \\
\text { escolares, em escola } \\
\text { pública }\end{array}$ & BREALD-30 & $\begin{array}{l}\text { Participantes com menor LSB foram } \\
\text { significativamente mais propensos a falhar ao } \\
\text { preencher questionários de pesquisa, com } \\
\text { questões abertas ou de múltipla escolha, } \\
\text { independente de escolaridade ou renda } \\
\text { familiar mensal }\end{array}$ & $\begin{array}{l}\text { Não foi realizado o ajuste } \\
\text { aprofundado do efeito da } \\
\text { educação na ausência dos } \\
\text { dados; Análise de apenas } \\
\text { uma habilidade relativa à } \\
\text { literácia }\end{array}$ \\
\hline $\begin{array}{l}\text { LIMA LCM, et al., 2019; } \\
\text { Estudo transversal }\end{array}$ & $\begin{array}{l}\text { Avaliar as propriedades } \\
\text { psicométricas do BREALD- } \\
30 \text { administrado para } \\
\text { adolescentes }\end{array}$ & $\begin{array}{l}750 \text { adolescentes } \\
\text { com } 12 \text { e } 15-19 \text { anos } \\
\text { de idade, em escolas } \\
\text { públicas e privadas }\end{array}$ & BREALD-30 & $\begin{array}{c}\text { A maioria dos adolescentes com baixo LSB } \\
\text { era de classe econômica menos privilegiada e } \\
\text { possuía pais com baixa escolaridade. Estudar } \\
\text { em escola pública foi associado ao menor } \\
\text { grau de LSB. Adolescentes com pontuações } \\
\text { do BREALD-30 mais baixas foram mais } \\
\text { propensos a apresentar lesões de cárie } \\
\text { cavitadas }\end{array}$ & $\begin{array}{c}\text { Análise de apenas uma } \\
\text { habilidade relativa à literácia }\end{array}$ \\
\hline $\begin{array}{l}\text { MONTES GR, et al., } \\
2019 \\
\text { Estudo transversal }\end{array}$ & $\begin{array}{l}\text { Avaliar a relação entre o } \\
\text { LSB de cuidadores e a cárie } \\
\text { não tratada de crianças pré- } \\
\text { escolares }\end{array}$ & $\begin{array}{l}415 \text { adultos } \\
\text { cuidadores e crianças } \\
\text { pré-escolares, em } \\
\text { escolas públicas }\end{array}$ & BREALD-30 & $\begin{array}{c}\text { A renda familiar foi maior em crianças livres } \\
\text { de cárie. O grau de LSB dos cuidadores foi } \\
\text { associado à presença de cárie não tratada e } \\
\text { infecção odontogênica em crianças. A } \\
\text { pontuação do BREALD-30, renda familiar, } \\
\text { escolaridade e estado civil foram associados } \\
\text { à cárie não tratada }\end{array}$ & $\begin{array}{l}\text { Análise de apenas uma } \\
\text { habilidade relativa à literácia; } \\
\text { Inclusão apenas de crianças } \\
\text { matriculadas em escolas } \\
\text { públicas }\end{array}$ \\
\hline $\begin{array}{l}\text { FIRMINO RT, et al., } \\
\quad 2019 ; \\
\text { Estudo transversal }\end{array}$ & $\begin{array}{l}\text { Validar a versão em } \\
\text { português brasileiro do } \\
\text { HKOHLAT-P }\end{array}$ & $\begin{array}{l}200 \text { adultos } \\
\text { cuidadores e crianças } \\
\text { pré-escolares, em } \\
\text { clínica odontológica }\end{array}$ & $\begin{array}{l}\text { BHKOHLAT-P } \\
\text { BREALD-30 }\end{array}$ & $\begin{array}{c}\text { A pontuação do BHKOHLAT-P demonstrou } \\
\text { correlação positiva significativa com a } \\
\text { escolaridade e horas de leitura }\end{array}$ & $\begin{array}{l}\text { A extensão do instrumento } \\
\text { pode afetar sua } \\
\text { aplicabilidade clínica; } \\
\text { Predominância de mulheres } \\
\text { respondentes }\end{array}$ \\
\hline
\end{tabular}

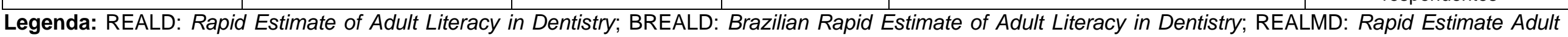

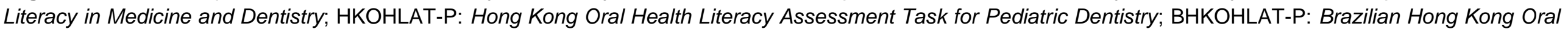
Health Literacy Assessment Task for Pediatric Dentistry.

Fonte: Lins RML, et al., 2020.

REAS / EJCH | Vol.12(12) | e4993 | DOI: https://doi.org/10.25248/reas.e4993.2020 Página 8 de 12 


\section{DISCUSSÃO}

Esta revisão integrativa identificou quatro instrumentos validados para o português do Brasil a fim de avaliar o grau de letramento em saúde bucal (LSB) em adultos, de forma que um destes também tenha demonstrado validade e confiabilidade para aplicação em adolescentes de 12 e 15 a 19 anos de idade.

Os resultados encontrados neste estudo indicam que pesquisas envolvendo o LSB no território brasileiro são recentes e demonstram tendência de expansão na comunidade científica, uma vez que os achados epidemiológicos têm relacionado as habilidades de letramento a importantes determinantes socioeconômicos e desfechos clínicos, contribuindo significativamente para a promoção de saúde, prevenção de doenças e aprimoramento de políticas de saúde bucal, mais direcionadas às individualidades de cada população.

Comum a todos os instrumentos, os autores do seu desenvolvimento original e da validação da sua versão brasileira não estabeleceram critérios para a categorização dos níveis de LSB como baixo, médio ou alto, fazendo com que os resultados obtidos sejam majoritariamente tratados como uma escala contínua (LEE JY, et al., 2007; GIRONDA M, et al., 2013; LEE J, et al., 2013; WONG HM, et al., 2013; JUNKES MC, et al., 2015; CRUVINEL AFP, et al., 2017; BADO FMR, et al., 2018; FIRMINO RT, et al., 2020).

Possivelmente, tal fato relaciona-se com a maior importância da identificação dos itens os quais o indivíduo demonstra dificuldade, quando comparado à sua simples agrupação em classificações, possibilitando ao clínico/pesquisador uma intervenção mais objetiva e individualizada para estratégias de educação em saúde.

O BREALD-30 demonstrou ser o instrumento mais empregado no país para a mensuração do LSB em estudos epidemiológicos, sendo também a ferramenta de escolha para a validação convergente de dois outros instrumentos (JUNKES MC, et al., 2015; CRUVINEL AFP, et al., 2017; FIRMINO RT, et al., 2020).

Tal método foi originalmente desenvolvido em inglês para a análise do LSB por intermédio do reconhecimento de 30 palavras relacionadas à rotina odontológica, abrangendo aspectos etiológicos, anatômicos, preventivos e curativos de condições bucais adversas (LEE JY, et al., 2007; VILELLA KD, et al., 2016). Durante a administração, é solicitada, ao entrevistado, a leitura em voz alta dos termos odontológicos, para que o examinador verifique a sua capacidade de pronunciação (JUNKES MC, et al., 2015).

A maioria dos artigos utilizando o BREALD-30 relatou como limitação da pesquisa a análise de apenas uma habilidade relativa à literácia em saúde (JUNKES MC, et al., 2015; VILELLA KD, et al., 2017; CRUVINEL AFP, et al., 2017; BADO FMR, et al., 2018; FIRMINO RT, et al., 2018; MONTES GR, et al., 2019; LIMA LCM, et al., 2019; FIRMINO RT, et al., 2020).

Tal fato sustenta-se na descontextualização das palavras apresentadas ao entrevistado, dispostas de forma aleatória para a leitura e, portanto, não permitindo ao examinador distinguir se o indivíduo compreende e é capaz de aplicar o item em seu cotidiano de maneira crítica ou se foi meramente apto a pronunciá-las. Assim, esse tipo de ferramenta para a mensuração do grau de LSB falha em avaliar o constructo em sua multidimensionalidade, embora possibilite uma administração fácil e rápida, bem como uma análise geral do nível de letramento do indivíduo (DICKSON-SWIFT V, et al., 2014; PARTHASARATHY DS, et al., 2014).

O REALMD-20 foi validado para o português brasileiro como uma alternativa de instrumento que reunisse termos médicos e odontológicos, baseado na presunção da indissociabilidade das áreas para a saúde integral do indivíduo (CRUVINEL AFP, et al., 2017).

A ferramenta, originalmente desenvolvida em inglês, possui o mesmo método de administração do BREALD-30, correspondendo ao reconhecimento de 20 palavras descontextualizadas a serem lidas em voz alta pelo entrevistado (GIRONDA M, et al., 2013).

Além da análise de apenas uma habilidade de letramento, Bado FMR et al. (2018) ressaltaram como lacuna metodológica do BREALD-30 e REALMD-20 o desenvolvimento original dos instrumentos na conjuntura linguística norte-americana, argumentando que a estrutura fonética altamente regular das línguas portuguesa e espanhola prejudica a equivalência e aplicabilidade de instrumentos traduzidos do inglês (LEE J, et al., 2013). 
Nesse contexto, o OHLA-B, originalmente desenvolvido em espanhol, foi validado para uso no Brasil com 15 itens referentes ao reconhecimento e compreensão de palavras (LEE J, et al., 2013; BADO FMR, et al., 2018). Seu método de administração é composto por duas etapas: inicialmente, é avaliada a pronunciação dos termos e, então, é solicitada ao entrevistado a escolha de associação do termo lido com dois outros, sendo um com significado clínico correlacionado e outro confundidor, sem vínculo com a palavra testada (STONBRAKER S, et al., 2015). Apesar da avaliação adicionar uma habilidade de letramento, este método ainda possui como limitação a análise restrita ao letramento funcional do indivíduo, não sendo capaz de identificar competências de numerácia e discernimento crítico (BADO FMR, et al., 2018).

O BHKOHLAT-P é a única ferramenta validada para uso no Brasil que avalia o grau de LSB de adultos específico para a saúde da criança, bem como a que apresenta o método mais robusto para tal análise (FIRMINO RT, et al., 2020). As habilidades examinadas são ordenadas em 49 itens, distribuídos por três sessões do instrumento, e englobam o conhecimento em saúde bucal pediátrica, compreensão de leitura e numerácia, aplicados em situações hipotéticas do cotidiano (WONG HM, et al., 2013; FIRMINO RT, et al., 2020). No entanto, a longa extensão e complexidade da ferramenta podem dificultar a sua aplicabilidade na rotina clínica, tornando a administração exaustiva ao entrevistado e custosa aos serviços de saúde.

Visto isso, todos os instrumentos validados para a mensuração do grau de LSB no Brasil dispõem de qualidades singulares e estão sujeitos à crítica metodológica. Tal fato reforça a necessidade da compreensão das propriedades psicométricas de cada método pelo examinador, para que a ferramenta mais adequada ao objetivo da sua pesquisa ou aplicação clínica seja selecionada.

A literatura científica aponta que sem a expansão da avaliação do LSB, os esforços políticos para a diminuição das disparidades presentes na saúde pública, avanço da prevenção primária, melhoria da qualidade dos serviços e redução dos custos de saúde não terão sucesso (HOROWITZ AM e KLEINMAN DV, 2012).

Em contrapartida, os instrumentos REALMD-20, OHLA-B e BHKOHLAT-P, apesar de indicarem potencialidades para pesquisas em saúde pública no Brasil, ainda não foram empregados em estudos epidemiológicos para além do processo de validação, impossibilitando a análise e comparação entre os resultados, assim como a avaliação da efetividade das ferramentas em território nacional (CRUVINEL AFP, et al., 2017; BADO FMR, et al., 2018; FIRMINO RT, et al., 2020).

Além disso, as pesquisas encontradas nesta revisão exibiram uma distribuição nacional destacadamente irregular, prejudicando a análise do grau de letramento em saúde bucal nos diferentes contextos socioeconômicos e demográficos do Brasil. A ausência de dados nas regiões Norte e Centro-Oeste, contraposta com a maior concentração dos estudos na região Sul $(60 \%)$, reflete a necessidade de uma disposição mais justa das investigações.

Considerando-se a desigualdade no que se refere à ordenação e acesso à educação e aos serviços de saúde pelo país, torna-se evidente a maior necessidade de mensuração do LSB em áreas de maior vulnerabilidade social, a fim de identificar falhas na comunicação profissional-paciente e refinar os meios de promoção de saúde e prevenção à doença, de acordo com as necessidades específicas e contexto socioeconômico e cultural de cada população (LANDMANN-SZWARCWALD C e MACINKO J, 2016; IBGE, 2019).

Outro ponto a ser julgado é a necessidade de futuros estudos epidemiológicos com amostragem aleatória em seu delineamento, a fim de avaliar o LSB em diferentes zonas e ser obtida uma representação mais fiel à estrutura demográfica brasileira. Isso porque a maioria dos estudos selecionados analisou o nível de LSB em serviços de saúde odontológicos ou hospitais universitários, fato que acarreta viés de seleção aos estudos devido à presunção de que indivíduos em ambientes clínicos usufruem de um maior acesso a informações de saúde, possivelmente superestimando o real nível de letramento da população local (JUNKES MC, et al., 2015; VILELLA KD, et al., 2016; CRUVINEL AFP, et al., 2017; BARASUOL JC, et al., 2017; VILELLA KD, et al., 2017; FIRMINO RT, et al., 2020). 
Dessa forma, pesquisas que utilizem os instrumentos recentemente validados para mensuração do grau de LSB são necessárias para que possa ser certificada a sua aplicabilidade em território nacional, dada a vasta influência da cultura local e propriedades linguísticas na efetividade da ferramenta. Futuros estudos epidemiológicos de avaliação do LSB no Brasil são fundamentais para que possam ser identificadas falhas nos métodos de educação em saúde atualmente empregados, aprimorando as políticas de saúde bucal e permitindo ao clínico/pesquisador uma intervenção mais próxima da realidade da população na qual atua.

Considerando tais recomendações, é possível pontuar certas limitações quanto à presente revisão. A breve introdução dos instrumentos em português brasileiro para a avaliação do LSB refletiu na restrita quantidade de estudos incluídos, fato que dificultou a análise crítica das suas propriedades psicométricas e dos resultados epidemiológicos obtidos. Além disso, devido à complexidade do constructo, a carência de métodos validados específicos para a mensuração qualitativa de cada ferramenta prejudicou a comparação das competências dos instrumentos de maneira padronizada. No entanto, a partir deste estudo, foi possível identificar lacunas epidemiológicas na distribuição geográfica e amostral das pesquisas encontradas, bem como particularizar as habilidades de cada ferramenta, a fim de auxiliar no direcionamento de futuros estudos no país e aprimorar os meios de educação em saúde bucal aplicados para cada população.

\section{CONSIDERAÇÕES FINAIS}

Os instrumentos para a mensuração do LSB validados para uso no Brasil apresentam propriedades psicométricas promissoras para a avaliação do alfabetismo em saúde bucal, sendo necessário que o clínico e/ou pesquisador compreenda as singularidades de cada ferramenta para que empregue a mais apropriada para o seu objetivo. Apesar da relevância da temática para as políticas de saúde bucal, os resultados encontrados nesta revisão demonstraram uma disposição demográfica desproporcional dos achados epidemiológicos em território nacional, de maneira que seja nítida a necessidade de expansão dos estudos para além dos ambientes clínicos, assim como alcançar regiões ainda não investigadas.

\section{REFERÊNCIAS}

1. IPM. Instituto Paulo Montenegro. 2018. In: Ação Social do IBOPE. Indicador de Analfabetismo Funcional (INAF). INAF Brasil 2018: Resultados preliminares. São Paulo. Disponível em: http://acaoeducativa.org.br/wpcontent/uploads/2018/08/Inaf2018_Relat\%C3\%B3rio-Resultados-Preliminares_v08Ago2018.pdf. Acesso em: 3 mar. 2020.

2. WHO. World Health Organization. 2013. In: Health literacy: the solid facts. Copenhagen: WHO Regional Office for Europe. Disponível em: https://apps.who.int/iris/bitstream/handle/10665/128703/e96854.pdf. Acesso em: 3 mar. 2020.

3. CARRAPATO P, et al. Determinante da saúde no Brasil: a procura da equidade na saúde. Saúde Soc. São Paulo, 2017; 26(3): 676-689.

4. WHO. World Health Organization. Promoting Health and Development: Closing the Implementation Gap. Proceedings of the $7^{\text {th }}$ Global Conference on Health Promotion. Nairobi, Kenya, 2009. Glob Health Promot, 2010; 17(2): 3-95.

5. BRASIL. Ministério da Saúde. Secretaria de Vigilância em Saúde. SB Brasil 2010: Pesquisa Nacional de Saúde Bucal: Resultados Principais. Brasília: SVS, 2012; 118p.

6. NATIONAL INSTITUTE OF DENTAL AND CRANIOFACIAL RESEARCH (NIDCR), et al. The invisible barrier: literacy and its relationship with oral health. A report of a workgroup sponsored by the National Institute of Dental and Craniofacial Research, National Institute of Health, U.S. Public Health Service, Department of Health and Human Services. J Public Health Dent, 2005; 65(3): 174-182.

7. SORENSEN K, et al. Health literacy and public health: a systematic review and integration of definitions and models. BMC Public Health, 2012; $12: 80$.

8. DICKSON-SWIFT V, et al. Measuring oral health literacy: a scoping review of existing tools. BMC Oral Health, 2014; 14: 148.

9. PARTHASARATHY DS, et al. Efficacy of instruments measuring oral health literacy: a systematic review. Oral Health Prev Dent, 2014; 12(3): 201-207.

10. WHITTEMORE R, KNAFL K. The integrative review: update methodology. J Adv Nurs, 2005; 52(5): 546-553.

11. HUTTON B, et al. The PRISMA extension statement for reporting of systematic reviews incorporating network metaanalyses of health care interventions: checklist and explanations. Ann Intern Med, 2015; 162(11): 777-784.

12. STONBRAKER S, et al. Tools to measure health literacy among Spanish speakers: An integrative review of the literature. Patient Educ Couns, 2015; 98(15): 1513-1523.

13. NUTBEAM D. The evolving concept of health literacy. Soc Sci Med, 2008; 67(12): 2072-2078. 
14. JUNKES MC, et al. Validity and Reliability of the Brazilian Version of the Rapid Estimate of Adult Literacy in Dentistry-BREALD-30. PLoS One, 2015; 10(7): e0131600.

15. CRUVINEL AFP, et al. The Brazilian version of the 20 -item rapid estimate of adult literacy in medicine and dentistry. PeerJ, 2017; 5: e3744.

16. BADO FMR, et al. Evaluation of the psychometric properties of the Brazilian version of the Oral Health Literacy Assessment in Spanish and development of a shortened form of the instrument. PLoS One, 2018; 13(11): e0207989.

17. FIRMINO RT, et al. Validation for Brazilian Portuguese language of the Hong Kong Oral Health Literacy Assessment Task for Paediatric Dentistry (BOHLAT-P). Int J Paed Dent, 2020; 30(2): 234-243.

18. MONTES GR, et al. Caregiver's oral health literacy is associated with prevalence of untreated dental caries in preschool children. Cien Saude Colet, 2019; 24(7): 2737-2744.

19. FIRMINO RT, et al. Impact of oral health literacy on self-reported missing data in epidemiological research. Community Dent Oral Epidemiol, 2018; 46(6): 624-630.

20. BARASUOL JC, et al. Oral Health Literacy as a Predictor of Dental Anxiety in Parents of Children Undergoing Dental Treatment. J Dent Child (Chic), 2017; 84(3): 125-131.

21. LIMA LCM, et al. Psychometric properties of BREALD-30 for assessing adolescents' oral health literacy. Rev Saude Publica, 2019; 53: 53.

22. VILELLA KD, et al. The Association of Oral Health Literacy and Oral Health Knowledge with Social Determinants in Pregnant Brazilian Women. J Community Health, 2016; 41(5): 1027-1032.

23. VILELLA KD, et al. Oral Health Literacy and Retention of Health Information Among Pregnant Women: A Randomised Controlled Trial. Oral Health Prev Dent, 2017; 15: 41-48.

24. LEE JY, et al. Development of a word recognition instrument to test health literacy in dentistry: the REALD-30 - A brief communication. J Public Health Dent, 2007; 67: 94-98.

25. GIRONDA M, et al. A brief 20 item dental/medical health literacy screen (REALMD-20). J Public Health Dent, 2013; 73(1): 50-55.

26. LEE J, et al. Oral Health Literacy Assessment: development of an oral health literacy instrument for Spanish speakers. J Public Health Dent, 2013; 73(1): 1-8.

27. WONG HM, et al. Validation of the Hong Kong Oral Health Literacy Assessment Task for Paediatric Dentistry (HKOHLAT-P). Int J Paediatr Dent, 2013; 23(5): 366-375.

28. VILELLA KD, et al. Training and calibration of interviewers for oral health literacy using the BREALD-30 in epidemiological studies. Braz Oral Res, 2016; 30(1): e90.

29. HOROWITZ AM, KLEINMAN DV. Oral health literacy: a pathway to reducing oral health disparities in Maryland. J Public Health Dent, 2012; 72(1): 26-30.

30. IBGE. Instituto Brasileiro de Geografia e Estatística. 2019. In: Síntese de Indicadores Sociais - Uma Análise das Condições de Vida da População Brasileira. Rio de Janeiro. Disponível em: https://biblioteca.ibge.gov.br/visualizacao/livros/liv101678.pdf. Acesso em: 3 de mar. 2020.

31. LANDMANN-SZWARCWALD C, MACINKO J. A panorama of health inequalities in Brazil. Int J Equity Health, 2016; 15: 174. 